\title{
Globulin, the albumin-to-globulin ratio, and fibrinogen perform well in the diagnosis of Periprosthetic joint infection
}

\author{
Huhu Wang ${ }^{\dagger}$, Haikang Zhou ${ }^{\dagger}$, Rendong Jiang, Zhenhao Qian, Fei Wang and Li Cao ${ }^{*}$
}

\begin{abstract}
Background: Although periprosthetic joint infection (PJI) is a severe complication of total joint arthroplasty (TJA), the diagnosis of PJI remains challenging. Albumin (ALB), globulin (GLB), the albumin-to-globulin ratio (AGR), and fibrinogen could be indicators of the body's inflammatory state. This study aimed to compare the diagnostic accuracy of these biomarkers with that of other inflammatory biomarkers in PJI patients.

Methods: We conducted a retrospective cohort study that included a consecutive series of patients undergoing debridement antibiotic irrigation and implant retention (DAIR), one-stage or the first stage of a two-stage revision total knee arthroplasty (TKA) or total hip arthroplasty (THA) for acute $(n=31)$ or chronic $(n=51)$ PJl, or revision TKA or THA for aseptic failures $(n=139)$ between January 2017 and December 2019 in our hospital. The 2013 criteria of the Musculoskeletal Infection Society (2013 MSIS) were used as the reference standard for the diagnosis of PJI. The preoperative ALB, GLB, AGR, fibrinogen, D-dimer, platelet count, fibrin degradation product (FDP), platelet-tolymphocyte (PLR), platelet count to mean platelet volume ratio (PVR), neutrophil-to-lymphocyte ratio (NLR), erythrocyte sedimentation rate (ESR) and C-reactive protein (CRP) levels were assessed. The receiver operating characteristic curve (ROC), sensitivity, and specificity were utilized to compare different biomarkers.

Results: Compared with the aseptic patients, the GLB, D-dimer, fibrinogen, FDP, platelet count, PVR, PLR, NLR, ESR, and CRP levels of PJl patients were significantly higher $(P<0.01)$; however, the ALB and AGR levels were significantly lower $(P<0.01)$. The area under the curve (AUC), sensitivity and specificity were $0.774,67.50,77.54 \%$ for ALB; $0.820,57.50,89.86 \%$ for GLB; $0.845,66.25,93.48 \%$ for AGR; $0.832,78.48,78.95 \%$ for fibrinogen; $0.877,81.48$, 85.07\% for ESR; 0.909, 83.95, 88.89\% for CRP; 0.683, 55.22, 75.83\% for D-dimer; $0.664,38.81,88.33 \%$ for FDP; 0.678, $52.44,79.86 \%$ for platelet count; $0.707,48.78,86.33 \%$ for PVR; $0.700,51.22,80.58 \%$ for PLR; and $0.678,52.44,81.30 \%$ for NLR, respectively. In the clinic, GLB, AGR and fibrinogen could be used for diagnosis of patients suspected of having PJI.
\end{abstract}

Conclusion: Our study demonstrated that GLB, AGR, and fibrinogen were promising biomarkers in the diagnosis of PJI.

Keywords: Periprosthetic joint infection, Diagnosis, Sensitivity, Specificity, Globulin, Albumin-to-globulin ratio, Fibrinogen

\footnotetext{
* Correspondence: xjbone@sina.com

${ }^{\dagger}$ Huhu Wang and Haikang Zhou contributed equally to this work. Department of Orthopaedics, First Affiliated Hospital of Xinjiang Medical University, Urumqi, Xinjiang, China
}

C C The Author(s). 2021 Open Access This article is licensed under a Creative Commons Attribution 4.0 International License, which permits use, sharing, adaptation, distribution and reproduction in any medium or format, as long as you give appropriate credit to the original author(s) and the source, provide a link to the Creative Commons licence, and indicate if changes were made. The images or other third party material in this article are included in the article's Creative Commons licence, unless indicated otherwise in a credit line to the material. If material is not included in the article's Creative Commons licence and your intended use is not permitted by statutory regulation or exceeds the permitted use, you will need to obtain permission directly from the copyright holder. To view a copy of this licence, visit http://creativecommons.org/licenses/by/4.0/ The Creative Commons Public Domain Dedication waiver (http://creativecommons.org/publicdomain/zero/1.0/) applies to the data made available in this article, unless otherwise stated in a credit line to the data. 


\section{Background}

Periprosthetic joint infection (PJI), a severe complication of total hip arthroplasty (THA) or total knee arthroplasty (TKA), is one of the major causes of joint revision surgery [1-4]. Furthermore, it has been reported that the incidence of PJI ranges between 2.0 and $2.4 \%$ for THA and TKA [5]. A timely and accurate diagnosis of PJI is essential to preserve the implanted prosthesis, restore adequate joint function, and even reduce the morbidity rate $[6,7]$. However, the diagnosis of PJI remains a challenge due to the lack of a "gold standard" and the fact that no currently available test is capable of absolute accuracy $[8,9]$.

The criteria provided by the Musculoskeletal Infection Society (MSIS) in 2011, modified in 2013, have been widely used in clinics $[10,11]$. Although the diagnostic criteria introduced by MSIS have undoubtedly dramatically improved the diagnosis of PJI, some individuals still fail to be diagnosed even after surgery [12]. Therefore, numerous diagnostic tests for new biomarkers and new molecular techniques have been evaluated [7, 13-18]. Among these new biomarkers, blood biomarkers may be attractive due to their convenience, especially for some routing tests administered to all inpatients [4, 18].

Albumin (ALB), one of the main components of serum proteins, is negatively interrelated with the inflammatory process $[19,20]$. Hypoalbuminemia, an index of malnutrition historically, has recently been a biomarker of inflammation [21, 22]. Serum globulin (GLB), such as components of complements and ceruloplasmin,

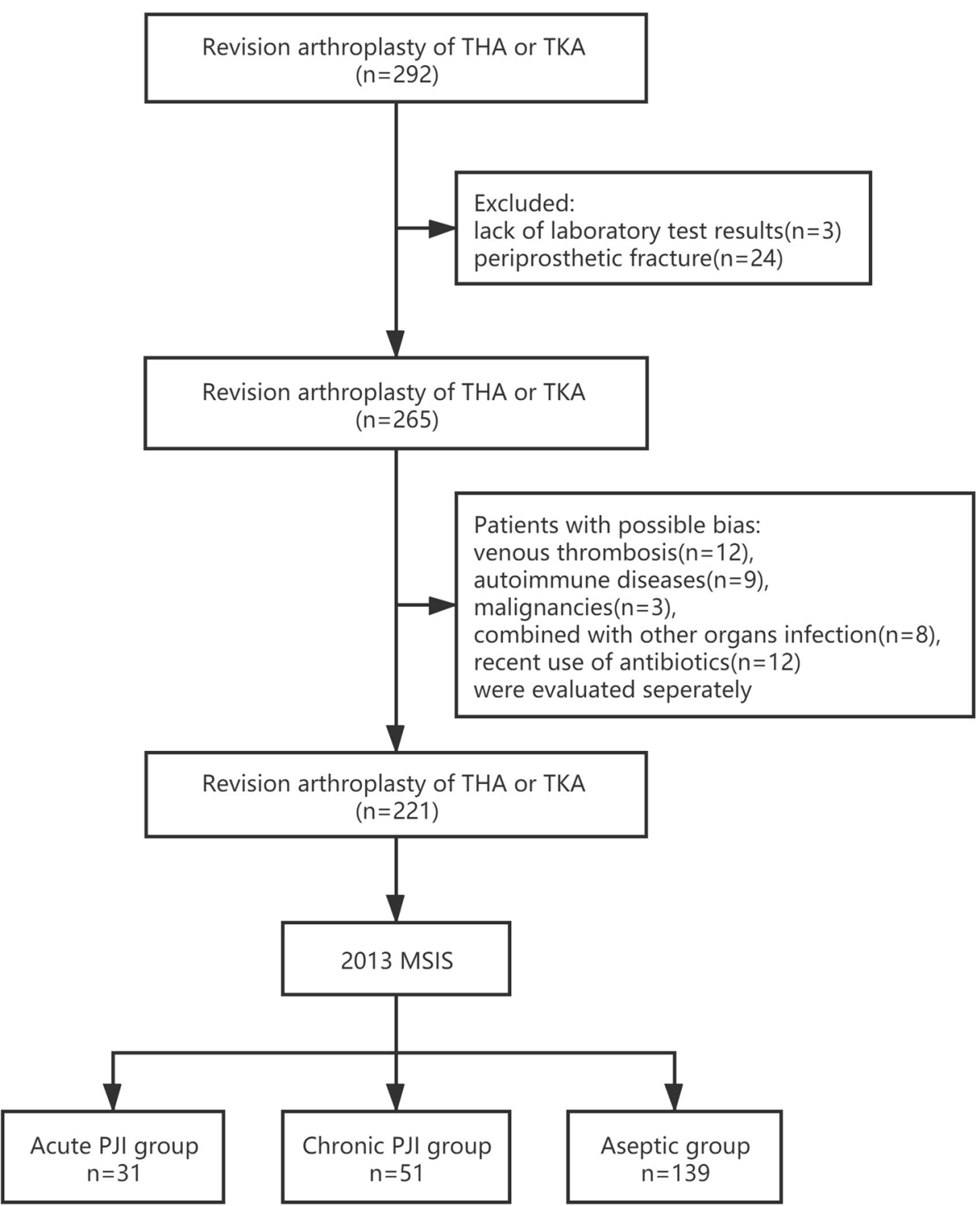

Fig. 1 Flowchart of the included and excluded patients in this study. PJl, periprosthetic joint infection; TKA, total knee arthroplasty; THA, total hip arthroplasty 
increases during the inflammatory process [23, 24]. Therefore, the albumin-to-globulin ratio (AGR), taking ALB and GLB into account, is a promising biomarker for inflammation $[25,26]$, but its application in detecting PJI has not been studied. D-dimer, fibrinogen, fibrin degradation product (FDP), and platelet count are new biomarkers of PJI, and they perform well in many studies $[8,27]$. Parvizi et al. [18] and Young-Min Kwon et al. [28] found that the platelet count to the mean platelet volume ratio (PVR) had specificity for PJI and could be used together with other biomarkers to improve the diagnostic performance of PJI. The neutrophil-tolymphocyte ratio (NLR) and the platelet-to-lymphocyte ratio (PLR) are simple biomarkers of inflammation in many diseases, such as hepatitis virus infection, rheumatic diseases, and infective endocarditis [29-31].

We performed this study to verify the PJI diagnostic accuracy of ALB, GLB, AGR, and fibrinogen. The assumption was that ALB, GLB, AGR, and fibrinogen would be promising biomarkers for PJI diagnosis. We compared these new biomarkers with other inflammatory biomarkers, namely, D-dimer, FDP, platelet count, PVR, PLR, NLR, erythrocyte sedimentation rate (ESR) and C-reactive protein (CRP).

\section{Methods}

\section{Study design and sample size estimation}

The sample size was calculated using PASS 11 software (NCSS). The incidence rate of PJI in TKA and THA revision surgery was estimated at $30 \%$. An area under the receiver operating characteristic (ROC) curve (AUC) of 0.75 was set for AUC 1, and an AUC of 0.6 was set for AUC 0 (floor level). A one-sided test was utilized to obtain a power (1-beta) of 0.9 and an $\alpha$ (significance level) of 0.01 . The loss ratio of follow-up was $10 \%$. Approximately 220 to 260 cases were recruited in this study. We conducted a retrospective cohort study that included a consecutive series of patients with painful hips or knees after total joint arthroplasty (TJA) who underwent debridement antibiotic irrigation and implant retention (DAIR), one-stage or the first stage of a two-stage revision TKA or THA for acute or chronic PJI or revision TKA or THA for aseptic failures in our hospital between January 2017 and December 2019. This study was approved by the Ethical Committee of our institution and informed consent was obtained in all cases before surgery was performed. Acute PJI was defined if the infection had been present for less than 3 months. The presence of PJI for more than 3 months was defined as chronic PJI. Some patients were excluded due to a lack of laboratory test results $(n=3)$ and periprosthetic fracture $(n=24)$. The cohort included 44 patients with acute PJI, 69 patients with chronic PJI, and 152 patients with aseptic failures. To rule out some possible bias, venous thrombosis $(n=12)$, autoimmune diseases $(n=9)$, malignancies $(n=3)$, concurrent infections in other organs $(n=8)$, and recent use of antibiotics 2 weeks before $(n=$ 12) were evaluated separately. Ultimately, 31 acute PJI patients were assigned to group A, 51 chronic PJI patients were assigned to group $\mathrm{B}$, and 139 aseptic failure patients were assigned to group C (Fig. 1). The basic demographic data are shown in Table 1 , and there was no significant difference in body mass index (BMI) between the PJI and aseptic failures. However, compared with aseptic failures, acute PJI patients' male proportion and age were greater $(P<0.05)$. Additionally, the PJI patients' knee revision rate was higher $(P<0.05)$.

\section{Diagnostic protocol and data extraction}

According to the institution's protocol, all patients who needed revision TKA or THA were well documented for their demographics, symptoms, signs, recent medicine use, and comorbidities in the electronic medical records system. Venous blood samples were collected by the

Table 1 Demographics of the groups

\begin{tabular}{|c|c|c|c|c|c|}
\hline & $\begin{array}{l}\text { Group A } \\
(=31)\end{array}$ & $\begin{array}{l}\text { Group B } \\
(n=51)\end{array}$ & $\begin{array}{l}\text { Group C } \\
(n=139)\end{array}$ & $\begin{array}{l}P \text { Valuet } \\
\text { (Group A vs. C) }\end{array}$ & $\begin{array}{l}P \text { Value } \\
\text { (Group B vs. C) }\end{array}$ \\
\hline Sex ${ }^{a}$ & & & & $0.043^{*}$ & 0.453 \\
\hline Male & 17 (54.8\%) & $21(41.2 \%)$ & 49 (35.3\%) & & \\
\hline Female & 14 (45.2\%) & 30 (58.8\%) & 90 (64.7\%) & & \\
\hline Age $(y)^{b}$ & 68 (60-74) & $68(53-74)$ & $63(49-71)$ & $0.031^{*}$ & 0.093 \\
\hline BMI $\left(\mathrm{kg} / \mathrm{m}^{2}\right)^{\mathrm{c}}$ & $25.26 \pm 3.55$ & $25.22 \pm 3.52$ & $25.49 \pm 4.12$ & 0.768 & 0.679 \\
\hline Involved Joint ${ }^{a}$ & & & & $0.043^{*}$ & $0.024^{*}$ \\
\hline Knee & 15 (48.4\%) & $24(47.1 \%)$ & 41 (29.5\%) & & \\
\hline Hip & 16 (51.6\%) & 27 (52.9\%) & 98 (70.5\%) & & \\
\hline
\end{tabular}

Group A, acute PJI group; Group B, chronic PJI group; Group C, aseptic failure group

${ }^{a}$ The values were given as number of cases and the percentage in parentheses

${ }^{b}$ Age was given as median with its interquartile range (25th - 75th) in parentheses

c $B M I$ were expressed as mean \pm SD. BMI, body mass index; SD, standard deviation

* $P<0.05$ 
nurses three to 7 days before surgery and were sent to the clinical laboratory within $2 \mathrm{~h}$. These blood samples were used to test routine blood examinations (including neutrophil count, lymphocyte count, platelet count, mean platelet volume), CRP, ESR, liver function tests (including ALB, GLB, AGR), and coagulation examinations (including D-dimer, fibrinogen, FDP). Joint aspiration was performed for the PJI suspicious patients, and synovial fluid was sent to test white blood cell (WBC) count and polymorphonuclear neutrophil percentage (PMN\%); at the same time, some synovial fluid was sent for aerobic and anaerobic cultures for 7-14 days. Additionally, at least three intraoperative samples were collected for aerobic and anaerobic cultures for 714 days. Histological analysis, including counting neutrophils per high-power field in 5 high-power fields, was routinely performed in all revision TKA or THA patients. All patients were followed up at least for 1 year. The 2013 criteria of the Musculoskeletal Infection Society (2013 MSIS), which is the most popular criterion, was used as the reference standard for the diagnosis of PJI [32]. Aseptic failures were defined as aseptic loosening, instability, wear, stiffness, implant breakage, unexplained pain, or aseptic reasons other than PJI. They did not fail because of infection within at least 1 year. All patients were followed for at least 1 year. All these data were well documented in our electronic medical records system and were carefully reviewed. Data extractors, follow-up investigators, and analyzers were blinded to the diagnosis of all patients.

\section{Laboratory assessments}

Routine blood examinations were performed on a Sysmex XE2100 hematology analyzer (Sysmex, Japan) in our hospital. CRP was measured through turbidimetric inhibition immunoassay (Beckman, America). ESR was measured using Westergren methods. Liver function tests were measured on a BACKMAN-LX 20 automatic biochemical analyzer (Backman, America). Coagulation was measured on a Sysmex CA-500 automatic blood coagulation analyzer (Sysmex, Japan).

\section{Statistical analysis}

Categorical variables were shown as counts (percentages), and the frequency distribution between the two groups was compared with the chi-square test. The comparisons of fibrinogen, ALB, GLB, AGR, platelet count, PVR, and BMI between the groups were performed using the independent $t$-test. The comparisons of D-dimer, FDP, PLR, NLR, ESR, CRP, and age between the two groups were analyzed using the Mann-Whitney $U$ test. ROC curves were plotted, and AUC was compared between different biomarkers. The optimal cutoff was determined by the Youden index. The sensitivity, specificity, positive predictive value (PPV), and negative predictive value (NPV) of each test were calculated. The AUC values were determined to be excellent $(0.900-1.000)$, good (0.800-0.899), fair (0.700-0.799), poor (0.600-0.699), and having no discriminatory capacity $(0.500-0.599)$ [13]. A biomarker was treated as a good marker if its AUC was greater than 0.8. Comparisons of good diagnostic biomarkers between hip and knee PJI

Table 2 Comparison of different biomarkers between PJI and aseptic groups

\begin{tabular}{|c|c|c|c|c|c|c|c|}
\hline Variables & Normal Rang & $\begin{array}{l}\text { Group A } \\
(n=31)\end{array}$ & $\begin{array}{l}\text { Group B } \\
(n=51)\end{array}$ & $\begin{array}{l}\text { Group C } \\
(n=139)\end{array}$ & $\begin{array}{l}P \text { Value } \\
\text { (A vs. B) }\end{array}$ & $\begin{array}{l}P \text { Value } \\
\text { (A vs. C) }\end{array}$ & $\begin{array}{l}P \text { Value } \\
\text { (B vs. C) }\end{array}$ \\
\hline$\overline{\mathrm{ALB}^{\mathrm{a}}(\mathrm{g} / \mathrm{L})}$ & $40-55$ & $35.33 \pm 3.67$ & $36.38 \pm 4.75$ & $40.29 \pm 3.72$ & 0.308 & $<0.001$ & $<0.001$ \\
\hline$G L B^{a}(g / L)$ & $20-40$ & $33.41 \pm 6.62$ & $33.16 \pm 5.40$ & $26.78 \pm 3.69$ & 0.858 & $<0.001$ & $<0.001$ \\
\hline$A G R^{a}$ & $1.2-2.4$ & $1.11 \pm 0.30$ & $1.13 \pm 0.28$ & $1.53 \pm 0.24$ & 0.731 & $<0.001$ & $<0.001$ \\
\hline$P\left\llcorner R^{b}\right.$ & - & $194.12(124.03-277.24)$ & $163.93(122.27-218.24)$ & 128.69 (103.49-157.34) & 0.308 & $<0.001$ & $<0.001$ \\
\hline$N L R^{b}$ & - & $2.53(1.83-3.40)$ & $2.26(1.62-3.21)$ & 1.75 (1.49-2.28) & 0.387 & $<0.001$ & 0.001 \\
\hline D-dimer ${ }^{\mathrm{b}}(\mathrm{ng} / \mathrm{ml})$ & $<280$ & 849 (256.5-1078.5) & 592.5 (322.5-833.75) & $331(189.5-576)$ & 0.344 & 0.003 & 0.001 \\
\hline Fibrinogen ${ }^{\mathrm{a}}(\mathrm{g} / \mathrm{L})$ & $2-4$ & $4.54 \pm 1.18$ & $4.41 \pm 0.81$ & $3.41 \pm 0.69$ & 0.572 & $<0.001$ & $<0.001$ \\
\hline $\mathrm{FDP}^{\mathrm{b}}(\mathrm{ug} / \mathrm{ml})$ & $0-5$ & $6.35(1.86-10.11)$ & 3.55 (2.48-5.78) & $2.75(1.6-3.8)$ & 0.273 & 0.007 & 0.002 \\
\hline Platelet count ${ }^{a}$ & $125-350$ & $300.74 \pm 92.34$ & $293.55 \pm 86.91$ & $243.22 \pm 65.33$ & 0.724 & 0.002 & $<0.001$ \\
\hline$P V R^{a}$ & - & $31.73 \pm 11.07$ & $30.54 \pm 11.09$ & $23.35 \pm 7.73$ & 0.639 & $<0.001$ & $<0.001$ \\
\hline $\mathrm{ESR}(\mathrm{mm} / \mathrm{h})^{\mathrm{b}}$ & $0-15$ & $55.00(40.00-66.50)$ & $50.00(40.00-60.00)$ & $21.50(13.75-32.50)$ & 0.332 & $<0.001$ & $<0.001$ \\
\hline $\operatorname{CRP}(\mathrm{mg} / \mathrm{L})^{\mathrm{b}}$ & $0-8$ & $25.75(12.28-52.28)$ & $20.30(11.50-31.40)$ & $3.59(2.23-6.31)$ & 0.371 & $<0.001$ & $<0.001$ \\
\hline
\end{tabular}

Group A, acute PJI group; Group B, chronic PJI group; Group C, aseptic failure group

${ }^{a}$ The values were given as mean \pm SD. SD, standard deviation

b The values were given as median with its interquartile range (25th - 75th) in parentheses

$P J l$ periprosthetic joint infection; $A L B$ albumin; GLB globulin; $A G R$ albumin-to-globulin ratio; PLR platelet-to-lymphocyte ratio; NLR neutrophil-to-lymphocyte ratio; FDP fibrin degradation product; PVR platelet count to mean platelet volume ratio; ESR erythrocyte sedimentation rate; $C R P$ C-reactive protein 
patients were analyzed. For patients with possible bias, including patients with venous thrombosis, autoimmune diseases, malignancies, and other organic infections, the recent use of antibiotics was evaluated separately based on the cutoff values of good diagnostic biomarkers. Culture results were shown, and good diagnostic biomarkers between positive and negative culture patients were analyzed. Analyses of diagnostic accuracy were exploratory. Statistical analysis and graphing were performed using SPSS version 24 (IBM Inc., Armonk, NY, USA) and GraphPad Prism software (Version 6; GraphPad Software Inc., San Diego, CA, USA). Statistical significance was set at $P<0.05$.

\section{Results}

Biomarker results

Compared with Group C, Groups A and B had significantly higher GLB, PLR, NLR, D-dimer, fibrinogen, FDP, platelet count, PVR, ESR, and CRP levels $(P<0.01$; Table 2; Fig. 2). Nonetheless, the ALB and AGR levels of Groups A and B were significantly lower than those of Group $C(P<0.01)$. These biomarkers were not significantly different between Groups A and B $(P>0.05)$.

Table 3 shows the AUCs, Youden index values, optimal cutoff values, and PJI predictive values (sensitivity, specificity, PPV, NPV) of the twelve biomarkers. The ROC curve (Fig. 3) analysis showed that the diagnostic performance of CRP was excellent (AUC $=0.909$ ), and

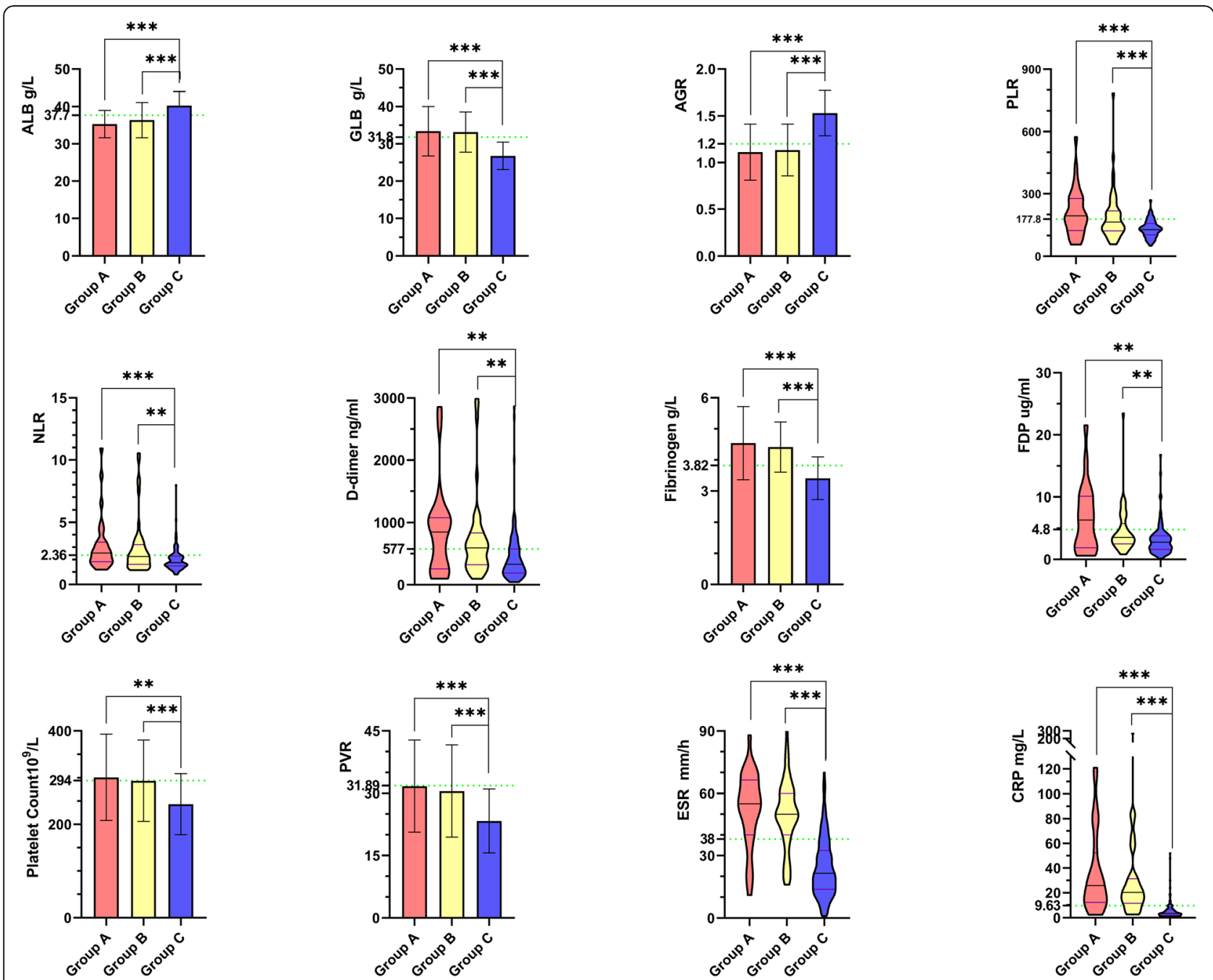

Fig. 2 The difference of ALB, GLB, AGR, fibrinogen, D-dimer, FDP, platelet count, PVR, PLR, NLR, ESR and CRP levels in acute PJI group, chronic PJI group and aseptic failure group. Group A, acute PJI group; Group B, chronic PJI group; Group C, aseptic failure group. PJI, periprosthetic joint infection; ALB, albumin; GLB, globulin; AGR, albumin-to-globulin ratio; PLR, platelet-to-lymphocyte ratio; NLR, neutrophil-to-lymphocyte ratio; FDP, fibrin degradation product; PVR, platelet count to mean platelet volume ratio; ESR, erythrocyte sedimentation rate; CRP, C-reactive protein. The data of ALB, GLB, AGR, fibrinogen, platelet count and PVR were shown as mean with standard deviation (SD), PLR, NLR, D-dimer, FDP, ESR and CRP were shown as median with interquartile range (25th-75th). The dashed line indicates the optimal cutoff values. ${ }^{* *} P<0.01 ;{ }^{* * *} P<0.001$ 
Table 3 Diagnostic performance of ALB, GLB, AGR, PLR, NLR, D-dimer, fibrinogen, FDP, platelet count, PVR, ESR and CRP for PJI

\begin{tabular}{|c|c|c|c|c|c|c|c|}
\hline & AUC $(95 \% \mathrm{Cl})$ & $\begin{array}{l}\text { Youden Index } \\
(95 \% \mathrm{Cl})\end{array}$ & $\begin{array}{l}\text { Optimal Cutoff } \\
(95 \% \mathrm{Cl})\end{array}$ & $\begin{array}{l}\text { Sensitivity(\%) } \\
(95 \% \mathrm{Cl})\end{array}$ & $\begin{array}{l}\text { Specificity(\%) } \\
(95 \% \mathrm{Cl})\end{array}$ & PPV(\%) $(95 \% \mathrm{Cl})$ & NPV(\%) $(95 \% \mathrm{Cl})$ \\
\hline ALB & $\begin{array}{l}0.774 \\
(0.713-0.828)\end{array}$ & $0.450(0.315-0.541)$ & $37.7(35.9-39.5)$ & $67.50(56.00-77.30)$ & $77.54(69.49-84.01)$ & $63.53(52.32-73.50)$ & $80.45(72.49-86.61)$ \\
\hline GLB & $0.820(0.763-0.869)$ & $\begin{array}{l}0.474 \\
(0.361-0.542)\end{array}$ & $31.8(29.4-34.1)$ & $57.50(45.95-68.32)$ & $\begin{array}{l}89.86 \\
(83.26-94.14)\end{array}$ & $76.67(63.66-86.22)$ & 78.48 (71.10-84.44) \\
\hline AGR & $0.845(0.790-0.891)$ & $0.597(0.468-0.690)$ & $1.2(1.16-1.46)$ & $66.25(54.72-76.21)$ & $93.48(87.62-96.78)$ & 85.48 (73.72-92.75) & $82.69(75.64-88.09)$ \\
\hline PLR & $\begin{array}{l}0.700 \\
(0.635-0.760)\end{array}$ & $0.376(0.242-0.465)$ & $177.8(146.8-214.1)$ & $51.22(40.01-62.32)$ & $80.58(72.82-86.60)$ & $60.87(48.35-72.17)$ & $73.68(65.81-80.33)$ \\
\hline NLR & $0.678(0.612-0.739)$ & $0.337(0.211-0.444)$ & $2.36(1.77-2.43)$ & $52.44(41.18-63.47)$ & $81.30(73.61-87.21)$ & $62.32(49.80-73.46)$ & $74.34(66.51-80.92)$ \\
\hline D-dimer & $0.683(0.612-0.749)$ & $0.311(0.160-0.427)$ & 577 (433-953) & $55.22(42.64-67.21)$ & $75.83(67.00-82.97)$ & $56.06(43.35-68.07)$ & $75.21(66.38-82.40)$ \\
\hline Fibrinogen & $0.832(0.775-0.880)$ & $0.574(0.446-0.665)$ & $3.82(3.75-4.27)$ & $\begin{array}{l}78.48 \\
(67.52-86.62)\end{array}$ & $78.95(70.85-85.34)$ & $68.89(58.14-78.00)$ & $86.07(78.35-91.44)$ \\
\hline FDP & $0.664(0.592-0.732)$ & $0.271(0.132-0.362)$ & $\begin{array}{l}4.8 \\
(2.3-6.5)\end{array}$ & $38.81(27.38-51.52)$ & $88.33(80.88-93.24)$ & $65.00(48.26-78.90)$ & $72.11(64.01-79.03)$ \\
\hline Platelet Count & $0.678(0.612-0.739)$ & $0.323(0.198-0.414)$ & $294(215.6-333.0)$ & $52.44(41.18-63.48)$ & $79.86(72.04-85.99)$ & $60.56(48.24-71.74)$ & $74.00(66.09-80.65)$ \\
\hline PVR & $0.707(0.642-0.766)$ & $0.351(0.230-0.453)$ & $31.89(27.37-40.71)$ & $48.78(37.68-59.99)$ & $86.33(79.23-91.36)$ & $67.80(54.24-79.03)$ & $74.07(66.50-80.49)$ \\
\hline ESR & $\begin{array}{l}0.877 \\
(0.825-0.917)\end{array}$ & $\begin{array}{l}0.866 \\
(0.547-0.752)\end{array}$ & $\begin{array}{l}38 \\
(34-44)\end{array}$ & $\begin{array}{l}81.48 \\
(70.98-88.93)\end{array}$ & $\begin{array}{l}85.07 \\
(77.65-90.43)\end{array}$ & $\begin{array}{l}76.74 \\
(66.16-84.89)\end{array}$ & $\begin{array}{l}88.37 \\
(81.25-93.13)\end{array}$ \\
\hline CRP & $\begin{array}{l}0.909 \\
(0.862-0.944)\end{array}$ & $\begin{array}{l}0.728 \\
(0.622-0.810)\end{array}$ & $\begin{array}{l}9.63 \\
(9.35-14)\end{array}$ & $\begin{array}{l}83.95 \\
(73.75-90.85)\end{array}$ & $\begin{array}{l}88.89 \\
(82.04-93.44)\end{array}$ & $\begin{array}{l}81.93 \\
(71.63-89.21)\end{array}$ & $\begin{array}{l}90.23 \\
(83.55-94.48)\end{array}$ \\
\hline
\end{tabular}

$\overline{A U C}$ area under the curve; $C l$ confidence interval; $P P V$ positive predictive value; $N P V$ negative predictive value; $P J /$ periprosthetic joint infection; $A L B$ albumin; $G L B$ globulin; $A G R$ albumin-to-globulin ratio; $P L R$ platelet-to-lymphocyte ratio; NLR neutrophil-to-lymphocyte ratio; FDP fibrin degradation product; $P V R$ platelet count to mean platelet volume ratio; ESR erythrocyte sedimentation rate; $C R P$ C-reactive protein

that those of ESR $(\mathrm{AUC}=0.877)$, AGR $(\mathrm{AUC}=0.845)$, fibrinogen $(\mathrm{AUC}=0.832)$ and $\mathrm{GLB}(\mathrm{AUC}=0.820)$ were good. The optimal cutoff value was based on the maximum Youden index. At the optimal cutoff value, the sensitivity, specificity, PPV and NPV were $83.95,88.89$, 81.93, $90.23 \%$ for CRP; $81.48,85.07,76.74,88.37 \%$ for ESR; $66.25 \%, 93.48,85.48,82.69 \%$ for AGR; $78.48,78.95$, $68.89,86.07 \%$ for fibrinogen; and $57.50,89.86,76.67$, $78.48 \%$ for GLB, respectively. However, other biomarkers' diagnostic performance levels were fair or poor, including ALB, PLR, PVR, NLR, D-dimer, FDP, and platelet count.

\section{Different joint results}

Table 4 shows the comparison between hip $(n=43)$ and knee $(n=39)$ PJI. There was no significant difference between hip and knee PJI in GLB (32.56 vs. 34.06, $P>0.05)$, AGR (1.16 vs. $1.09, P>0.05$ ), fibrinogen (4.37 vs. 4.56, $P>0.05$ ), ESR (50.0 vs. $54.0, P>0.05$ ), and CRP (20.3 vs. $22.9, P>0.05$ ). Based on the optimal threshold, the diagnostic accuracies of GLB, AGR, fibrinogen, ESR, and CRP were $55.81,67.44,81.40,81.40,86.05 \%$, respectively, in hip PJI, while the diagnostic accuracies of GLB, AGR, fibrinogen, ESR, and CRP were 61.54, 66.67, 79.49, 79.49, 79.49\%, respectively, in knee PJI.

\section{Comorbidities analysis}

In particular, we evaluated the diagnostic performance of GLB, AGR, fibrinogen, ESR, and CRP in patients with venous thrombosis, autoimmune diseases, malignancies, and concurrent infections in other organs based on the optimal threshold (Table 5). The diagnostic accuracies of ALB, AGR, fibrinogen, and ESR were nearly identical (75\%) and lower than that of CRP (91.67\%) in patients with venous thrombosis. The diagnostic accuracies of fibrinogen and CRP were the same (77.78\%) and
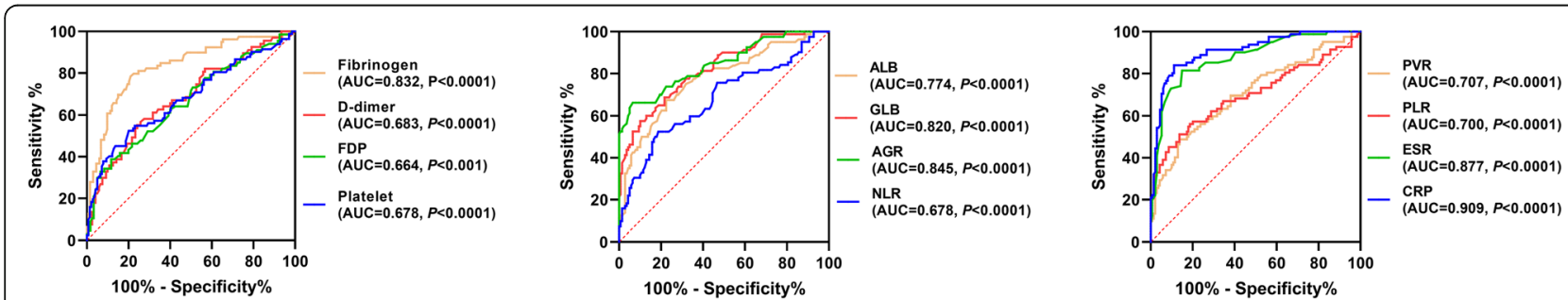

Fig. 3 The ROC curves and AUCs of ALB, GLB, AGR, fibrinogen, D-dimer, FDP, platelet count, PVR, PLR, NLR, ESR and CRP. ROC, receiver operating characteristic curve; AUC, area under the curve; ALB, albumin; GLB, globulin; AGR, albumin-to-globulin ratio; PLR, platelet-to-lymphocyte ratio; NLR, neutrophil-to-lymphocyte ratio; FDP, fibrin degradation product; PVR, platelet count to mean platelet volume ratio; ESR, erythrocyte sedimentation rate; CRP, C-reactive protein 
Table 4 Comparison of GLB, AGR, fibrinogen, ESR and CRP between hip and knee PJI

\begin{tabular}{|c|c|c|c|c|c|}
\hline Variables & Normal Rang & Hip PJI $(n=43)$ & Knee PJI $(n=39)$ & Statistics Test & $P$ Value \\
\hline$\overline{G L B}(g / L)^{a}$ & $20-40$ & $32.56 \pm 5.52$ & $34.06 \pm 6.15$ & $t=-1.152$ & 0.253 \\
\hline$A G R^{a}$ & $1.2-2.4$ & $1.16 \pm 0.28$ & $1.09 \pm 0.29$ & $t=0.973$ & 0.333 \\
\hline Fibrinogen $(\mathrm{g} / \mathrm{L})^{\mathrm{a}}$ & $2-4$ & $4.37 \pm 0.80$ & $4.56 \pm 1.10$ & $t=0.870$ & 0.387 \\
\hline $\operatorname{ESR}(\mathrm{mm} / \mathrm{h})^{\mathrm{b}}$ & $0-15$ & $50.0(40.0-60.0)$ & $54.0(40.0-62.5)$ & $Z=-0.526$ & 0.599 \\
\hline $\operatorname{CRP}(\mathrm{mg} / \mathrm{L})^{\mathrm{b}}$ & $0-8$ & $20.3(11.2-30.9)$ & $22.9(12.6-56.9)$ & $Z=-0.804$ & 0.421 \\
\hline
\end{tabular}

GLB globulin; $A G R$ albumin-to-globulin ratio; ESR erythrocyte sedimentation rate; CRP C-reactive protein

${ }^{a}$ The values were given as mean \pm SD. SD, standard deviation

b The values were given as median with its interquartile range (25th - 75th) in parentheses

outstanding compared to others in patients with combined autoimmune diseases. The diagnostic accuracies of GLB and fibrinogen were equal to (100\%) and better than those of other markers $(66.67 \%)$ in patients with combined malignancies. Nevertheless, for patients with concurrent infections in other organs, the accuracies of GLB, AGR, and CRP all achieved 100\%.

\section{Recent use of antibiotics results}

Table 6 shows the comparison between PJI patients $(n=$ 12) who used antibiotics 2 weeks before and PJI patients $(n=82)$ who had not used antibiotics recently. There were no significant differences between the cases of PJI in patients who had recently used and had not used antibiotics in terms of GLB (33.87 vs. $33.25, P>0.05)$, AGR (1.20 vs. $1.13, P>0.05)$, fibrinogen (4.45 vs. $4.46, P>$ $0.05)$, ESR (50.0 vs. $52.0, P>0.05)$, and CRP (36.3 vs. 21.3, $P>0.05$ ).

\section{Culture results}

Table 7 shows the culture results of PJI patients. Among the 82 PJI patients, 59 achieved positive cultures (71.95\%), and the two most common pathogens were Staphylococcus epidermidis (33.90\%, 20 of 59) and Staphylococcus aureus (18.64\%, 11 of 59). The positive culture rate $(66.67 \%, 8$ of 12$)$ in the twelve PJI patients with antibiotics recently was lower than that in PJI patients not treated with antibiotics $(71.95 \%, 59$ of 82$)$. There were no significant differences between the culture-positive and culture-negative PJI patients in terms of GLB (33.97 vs. $31.23, P>0.05)$, AGR (1.09 vs. $1.22, P>0.05)$, fibrinogen (4.52 vs. $4.30, P>0.05)$, and CRP (21.7 vs. 20.3, $P>0.05)$ (Table 8). The ESR of positive culture PJI was significantly higher than that of negative culture PJI (54.0 vs. 46.0, $P<0.05$ ). Based on the optimal threshold, the diagnostic accuracies of GLB, AGR, fibrinogen, ESR, and CRP were 62.71, 71.19, 83.05, 83.05 , and $89.83 \%$, respectively, in culture-positive PJI and $47.83,56.52,73.91,73.91$, and $65.22 \%$, respectively, in culture-negative PJI.

\section{Discussion}

Based on a literature review, this study was the first to evaluate the diagnostic performances of ALB, GLB, and AGR in differentiating PJI and aseptic failure. We compared ALB, GLB, and AGR with some inflammatory biomarkers (D-dimer, fibrinogen, FDP, platelet count, PVR, PLR, NLR, ESR, and CRP). We found that GLB, AGR, fibrinogen, ESR, and CRP had good performance in the diagnosis of PJI. At the optimal cutoff point of AGR, apart from its low sensitivity (66.25\%), the specificity (93.48\%) was the highest among these biomarkers, reducing the misdiagnosis rate of PJI. Additionally, the PPV of AGR (85.48\%) was the highest; that is, AGR was superior in predicting the diagnosis of PJI. The diagnostic value of fibrinogen was slightly lower than that of AGR based on the AUC analysis. However, the diagnostic value of ALB was fair, with an AUC, sensitivity, and specificity of $0.774,67.50$, and $77.54 \%$, respectively. In terms of this study, GLB, and AGR were good biomarkers; they were two essential markers in liver function tests that were routinely performed before surgery with no time wasted and no further cost. Hence, GLB and AGR are two promising and adequate biomarkers to aid in diagnosing PJI.

Table 5 Diagnostic performance of GLB, AGR, fibrinogen, ESR and CRP for patients with some comorbidities

\begin{tabular}{|c|c|c|c|c|c|c|c|c|c|c|}
\hline \multirow{2}{*}{$\begin{array}{l}\text { Comorbidities and } \\
\text { Numbers }\end{array}$} & \multicolumn{2}{|l|}{ GLB } & \multicolumn{2}{|l|}{ AGR } & \multicolumn{2}{|c|}{ Fibrinogen } & \multicolumn{2}{|l|}{ ESR } & \multicolumn{2}{|l|}{ CRP } \\
\hline & PJ & Aseptic & PJI & Aseptic & PJI & Aseptic & PJI & Aseptic & PJI & Aseptic \\
\hline Venous Thrombosis $(n=12)$ & $4(2)$ & $8(7)$ & $4(2)$ & $8(7)$ & $4(1)$ & $8(7)$ & $4(2)$ & $8(7)$ & $4(3)$ & $8(8)$ \\
\hline Autoimmune Diseases $(n=9)$ & $6(2)$ & $3(3)$ & $6(3)$ & $3(3)$ & $6(5)$ & $3(2)$ & $6(3)$ & $3(2)$ & $6(4)$ & $3(3)$ \\
\hline Malignancies $(n=3)$ & $1(1)$ & $2(2)$ & $1(1)$ & $2(1)$ & $1(1)$ & $2(2)$ & $1(1)$ & $2(1)$ & $1(1)$ & $2(1)$ \\
\hline Concurrent Infections $(n=8)$ & $8(7)$ & 0 & $8(7)$ & 0 & $8(6)$ & 0 & $8(6)$ & 0 & $8(7)$ & 0 \\
\hline
\end{tabular}

GLB globulin; AGR albumin-to-globulin ratio; ESR erythrocyte sedimentation rate; CRP C-reactive protein 
Table 6 Comparison of GLB, AGR, fibrinogen, ESR and CRP between the PJl patients who used antibiotics two weeks before and PJI patients who had not used antibiotics recently

\begin{tabular}{llllcc}
\hline Variables & Normal Rang & $\begin{array}{l}\text { PJI Recent Use of } \\
\text { Antibiotics }(\boldsymbol{n}=\mathbf{1 2})\end{array}$ & $\begin{array}{l}\text { PJ No Use of } \\
\text { Antibiotics }(\boldsymbol{n}=\mathbf{8 2})\end{array}$ & Statistics Test & $\boldsymbol{P}$ Value \\
\hline GLB $(\mathrm{g} / \mathrm{L})^{\mathrm{a}}$ & $20-40$ & $33.87 \pm 10.62$ & $33.25 \pm 5.83$ & $t=0.197$ & 0.847 \\
AGR $^{\mathrm{a}}$ & $1.2-2.4$ & $1.20 \pm 0.58$ & $1.13 \pm 0.28$ & $t=0.451$ & 0.660 \\
Fibrinogen $(\mathrm{g} / \mathrm{L})^{\mathrm{a}}$ & $2-4$ & $4.45 \pm 1.16$ & $4.46 \pm 0.95$ & $t=-0.019$ & 0.985 \\
ESR $(\mathrm{mm} / \mathrm{h})^{\mathrm{b}}$ & $0-15$ & $50.0(37.8-58.5)$ & $52.0(40.0-61.5)$ & $Z=-0.459$ & 0.646 \\
CRP $(\mathrm{mg} / \mathrm{L})^{\mathrm{b}}$ & $0-8$ & $36.3(7.7-49.0)$ & $21.3(12.3-35.3)$ & $Z=-0.453$ & 0.651 \\
\hline
\end{tabular}

GLB globulin; $A G R$ albumin-to-globulin ratio; $E S R$ erythrocyte sedimentation rate; CRP C-reactive protein

a The values were given as mean \pm SD. SD, standard deviation

b The values were given as median with its interquartile range (25th - 75th) in parentheses

Albumin is typically utilized to evaluate nutritional status; recent studies have demonstrated that albumin is a negative phase reactant, as its synthesis decreases during inflammation [33-35]. Previous research conducted by Miraeidi $M$ et al. [22] found that inflammation could reduce albumin levels irrespective of the patients' nutritional state. One possible explanation of this phenomenon was that inflammatory mediators could promote albumin escape from blood capillaries and reprioritize hepatic protein synthesis in favor of acute-phase reactants [36]. Globulin consists of many proteins associated with inflammation, such as complements, interleukin-6, and immunoglobulins; thus, elevated globulin reflects an inflammatory state $[20,37,38]$.

Table 7 Culture results of PJI patients $(n=82)$

\begin{tabular}{ll}
\hline Culture Results & Number of patients \\
\hline Positive & 59 \\
Staphylococcus epidemidis & 20 \\
Staphylococcus aureus & 11 \\
Staphylococcus capitis & 3 \\
Brucella & 2 \\
Pseudomonas aeruginosa & 2 \\
Enterobacter cloacae & 2 \\
Streptococcus mitis & 2 \\
Streptococcus sanguis & 2 \\
Proteus mirabilis & 1 \\
Bacillus subtilis & 1 \\
Bacillus firmus & 1 \\
Human Staphylococcus & 1 \\
Staphylococcus lugdunensis & 1 \\
Staphylococcus haemolyticus & 1 \\
Canidia albicans & 1 \\
Staphylococcus saprophyticus & 1 \\
Escherichia coli & 1 \\
Polymicrobial & 6 \\
Negative & 23 \\
\hline
\end{tabular}

As both decreased albumin and increased globulin played essential roles in inflammation, the AGR, which took albumin and globulin into account concurrently, could indicate the body's inflammatory state more accurately $[20,26,39,40]$.

Coagulation-associated biomarkers are usually used to monitor venous thromboembolism and assess coagulation status before surgery. Recently, many studies have demonstrated that inflammation is closely related to the coagulation cascade [41, 42]. D-dimer, fibrinogen, and FDP were promising biomarkers for the diagnosis of PJI in some studies $[8,27]$. In this study, fibrinogen's diagnostic performance was good, and the results of sensitivity, specificity, and AUC were similar to Li et al.'s study [43]. However, the diagnostic values of D-dimer and FDP were limited, and this result was proven by $\mathrm{Xu} \mathrm{H}$ et al.'s study [44]. In the clinic, fibrinogen could be used for diagnosis in patients suspected of PJI.

Gaertner $\mathrm{F}$ et al. [45] demonstrated the anti-infection role of platelets in collecting and bundling bacterial functions. Parvizi et al. [18] showed that PVR increased in PJI patients, and its diagnostic sensitivity, specificity, and AUC were $48.10,80.85 \%$, and 0.69 , respectively, similar to the findings of this study. Compared with GLB, AGR, and fibrinogen, the diagnostic value of platelet count and PVR was limited.

PLR and NLR are simple biomarkers of inflammation. Previous research conducted by Zhao Z et al. [29] found that PLR and NLR were associated with chronic hepatitis $B$ virus infection. In addition, Gasparyan A. Y. et al. [30] found that PLR could be an inflammatory marker in rheumatic diseases. In this study, we found that the diagnostic performance of PLR and NLR was limited, as the AUC, sensitivity and specificity of PLR were 0.700 , $51.22,80.58 \%$, respectively, and those of NLR were $0.678,52.44$ and $81.30 \%$, respectively.

In the analyses of different joints, we found that the GLB, AGR, fibrinogen, ESR, and CRP levels between hip and knee PJI patients were nearly the same. Furthermore, the diagnostic accuracies of these biomarkers were not largely different. 
Table 8 Comparison of GLB, AGR, fibrinogen, ESR, and CRP between culture-positive and culture-negative PJI

\begin{tabular}{llllll}
\hline Variables & Normal Rang & Culture-Positive PJI $(\boldsymbol{n}=\mathbf{3 7})$ & Culture-Negative PJI $(\boldsymbol{n}=14)$ & Statistics Test & $\boldsymbol{P}$ Value \\
\hline GLB $(\mathrm{g} / \mathrm{L})^{\mathrm{a}}$ & $20-40$ & $33.97 \pm 5.53$ & $31.23 \pm 6.32$ & $t=1.881$ & 0.064 \\
AGR $^{\mathrm{a}}$ & $1.2-2.4$ & $1.09 \pm 0.26$ & $1.22 \pm 0.33$ & $t=-1.614$ & 0.117 \\
Fibrinogen $(\mathrm{g} / \mathrm{L})^{\mathrm{a}}$ & $2-4$ & $4.52 \pm 1.02$ & $4.30 \pm 0.73$ & $t=0.878$ & 0.382 \\
ESR $(\mathrm{mm} / \mathrm{h})^{\mathrm{b}}$ & $0-15$ & $54.0(46.0-64.3)$ & $46.0(26.0-54.0)$ & $Z=-2.371$ & 0.018 \\
$\mathrm{CRP}(\mathrm{mg} / \mathrm{L})^{\mathrm{b}}$ & $0-8$ & $21.7(13.9-35.0)$ & $20.3(6.5-49.2)$ & $Z=-1.330$ & 0.183 \\
\hline
\end{tabular}

GLB globulin; $A G R$ albumin-to-globulin ratio; ESR erythrocyte sedimentation rate; $C R P$ C-reactive protein

${ }^{a}$ The values were given as mean \pm SD. SD, standard deviation

b The values were given as median with its interquartile range (25th -75 th) in parentheses

We evaluated the diagnostic accuracies of GLB, AGR, fibrinogen, ESR, and CRP in patients with comorbidities (venous thrombosis, autoimmune diseases, malignancies, and concurrent infections in other organs) separately and found that their diagnostic accuracies were good. As the number of cases was small, the diagnostic accuracies of these biomarkers should be evaluated in a larger number of cases in the future.

The recent use of antibiotics might influence inflammation biomarkers. However, in this study, we found no significant difference between PJI patients who recently used and did not recently use antibiotics in GLB, AGR, fibrinogen, ESR, and CRP. Therefore, the use of antibiotics had little influence on these biomarkers, and it was attractive to use these three biomarkers in the PJI diagnosis of patients who had recently used antibiotics.

Benito $\mathrm{N}$ et al. [46] conducted a multicenter cohort study of PJI and obtained 2288 cases with the microbiologic diagnosis. In their research, Staphylococcus aureus, Staphylococcus epidermidis, Escherichia coli, Pseudomonas aeruginosa, Enterococcus faecalis, and Propionibacterium acnes, in decreasing order, were involved in more than $80 \%$ of PJIs. In our study, Staphylococcus epidermidis was the most common pathogen $(33.90 \%$, 20 of 59), followed by Staphylococcus aureus (18.64\%, 11 of 59). This difference was caused by the bacterial spectrum varying among different areas. There was no significant difference between the culture-positive and culture-negative PJI patients in GLB, AGR, fibrinogen, and CRP. However, the diagnostic accuracies of these biomarkers in culture-negative PJI were lower than those of culture-positive PJI: GLB (47.83\% vs. $62.71 \%)$, AGR (56.52\% vs. $71.19 \%)$, fibrinogen $(73.91 \%$ vs. $83.05 \%)$, and CRP (65.22\% vs. $89.83 \%)$. Therefore, more attention is needed in the diagnosis of culture-negative PJI. The positive culture rate of PJI patients with recent use of antibiotics was lower than that of those who had not recently used antibiotics; thus, the use of antibiotics decreased the culture rate.

There are some limitations to this study. The most important limitation related to this study is the retrospective nature. Another limitation is the small sample size of this study, necessitating further large-scale, prospective, and multicenter studies. Finally, this study lacks validation, which may lead to the reduced reliability of these new biomarkers; we will verify this hypothesis in future studies.

\section{Conclusion}

Our study demonstrated that GLB, AGR, and fibrinogen were promising biomarkers in the diagnosis of PJI.

\section{Abbreviations}

BMI: body mass index; MSIS: Musculoskeletal infection society; PJl: Periprosthetic joint infection; THA: Total hip arthroplasty; TKA: Total knee arthroplasty; TJA: Total joint arthroplasty; DAIR: Debridement antibiotic irrigation and implant retention; ALB: Albumin; GLB: globulin; AGR: Albuminto-globulin ratio; PLR: Platelet-to-lymphocyte ratio; NLR: Neutrophil-tolymphocyte ratio; FDP: Fibrin degradation product; PVR: Platelet count to mean platelet volume ratio; ESR: Erythrocyte sedimentation rate; CRP: CReactive protein; WBC: White blood cell; PMN: Polymorphonuclear neutrophil; SD: Standard deviation; ROC: Receiver operating characteristic; AUC: Area under the curve; Cl: Confidence interval; PPV: Positive predictive value; NPV: Negative predictive value

\section{Acknowledgments}

Not applicable.

\section{Authors' contributions}

HHW conceived the idea and wrote framework and manuscript of the paper. HKZ designed the study and edited the manuscript. RDJ performed the statistical analysis. ZHQ carried out interpretation of data and manuscript preparation. FW collected, sorted and interpreted the data. LC planned the project and critically revised the paper. All autors read and approved the final manuscript.

\section{Funding}

The National Key Research and Development Projects of China (No.2017YFB1304204) supported the design, writing, collection, analysis, and follow-up tasks related to this manuscript.

\section{Availability of data and materials}

The data used and analyzed during the current study are available from the corresponding author on reasonable request.

\section{Declarations}

Ethics approval and consent to participate

Approval was obtained from the institutional ethics committee of the First Affiliated Hospital of Xinjiang Medical University (IRB number: K202010-10). No administrative permissions were required to access and use the medical records described in our study. All patients signed an informed consent form.

Consent for publication

Not applicable. 


\section{Competing interests}

All authors declare that they have no conflict of interest.

Received: 22 December 2020 Accepted: 7 June 2021 Published online: 25 June 2021

\section{References}

1. Shahi A, Parvizi J. The role of biomarkers in the diagnosis of periprosthetic joint infection. EFORT Open Rev. 2016;1 (7):275-8. https://doi.org/10.1302/2 058-5241.1.160019.

2. Koh CK, Zeng I, Ravi S, Zhu M, Vince KG, Young SW. Periprosthetic joint infection is the Main cause of failure for modern knee arthroplasty: an analysis of 11,134 knees. Clin Orthop Relat Res. 2017;475(9):2194-201. https://doi.org/10.1007/s1 1999-017-5396-4.

3. Cozzi LA, Del PA, Soderi S, Innocenti M, Civinini R. The identification of pathogens associated with periprosthetic joint infection in two-stage revision. Eur Rev Med Pharmacol Sci. 2019;23(2 Suppl):101-16.

4. Schwartz AM, Farley KX, Guild GN, Bradbury TJ. Projections and epidemiology of revision hip and knee arthroplasty in the United States to 2030. J Arthroplast. 2020;35(6S):S79-85. https://doi.org/10.1016/.arth.2 020.02.030.

5. Kurtz SM, Lau E, Watson H, Schmier JK, Parvizi J. Economic burden of periprosthetic joint infection in the United States. J Arthroplast. 2012;27(8 Suppl):61-5. https://doi.org/10.1016/j.arth.2012.02.022.

6. Fehring TK, Odum SM, Berend KR, Jiranek WA, Parvizi J, Bozic KJ, et al. Failure of irrigation and debridement for early postoperative periprosthetic infection. Clin Orthop Relat Res. 2013;471(1):250-7. https://doi.org/10.1007/ s11999-012-2373-9.

7. Marazzi MG, Randelli F, Brioschi M, Drago L, Romano CL, Banfi G, et al. Presepsin: a potential biomarker of PJI? A comparative analysis with known and new infection biomarkers. Int J Immunopathol Pharmacol. 2018;31: 1832658284.

8. Wang Y, Li Y, Qiao L, Sun S. Comparison of a comprehensive set of fibrinolytic markers with C-reactive protein and erythrocyte sedimentation rate for the diagnosis of Periprosthetic joint infection. J Arthroplast. 2020; 35(9):2613-8. https://doi.org/10.1016/j.arth.2020.04.096.

9. Fernandez-Sampedro M, Farinas-Alvarez C, Garces-Zarzalejo C, AlonsoAguirre MA, Salas-Venero C, Martinez-Martinez L, et al. Accuracy of different diagnostic tests for early, delayed and late prosthetic joint infection. BMC Infect Dis. 2017;17(1):592. https://doi.org/10.1186/s12879-017-2693-1.

10. New definition for periprosthetic joint infection. J ARTHROPLASTY 2011, 26(8):1136-1138.

11. Parvizi J, Gehrke T, Chen AF. Proceedings of the international consensus on Periprosthetic joint infection. Bone Joint J. 2013;95-B(11):1450-2. https://doi. org/10.1302/0301-620X.95B11.33135.

12. Parvizi J, Tan TL, Goswami K, Higuera C, Della VC, Chen AF, et al. The 2018 definition of Periprosthetic hip and knee infection: an evidence-based and validated criteria. J Arthroplast. 2018;33(5):1309-14. https://doi.org/10.1016/ j.arth.2018.02.078.

13. Bin $G$, Xinxin $Y$, Fan $L$, Shenghong $W$, Yayi X. Serum fibrinogen test performs well for the diagnosis of Periprosthetic joint infection. J Arthroplast. 2020;35(9):2607-12. https://doi.org/10.1016/j.arth.2020.04.081.

14. Kim SG, Kim JG, Jang KM, Han SB, Lim HC, Bae JH. Diagnostic value of synovial white blood cell count and serum C-reactive protein for acute Periprosthetic joint infection after knee arthroplasty. J Arthroplast. 2017; 32(12):3724-8. https://doi.org/10.1016/j.arth.2017.07.013.

15. Busch A, Jager M, Engler H, Haversath M, Bielefeld C, Landgraeber S, et al. Is Procalcitonin (PCT) a reliable biomarker for preoperative diagnosing of low grade periprosthetic joint infection? A prospective study. BMC Musculoskelet Disord. 2020;21(1):257. https://doi.org/10.1186/ s12891-020-03266-6.

16. Wang C, Wang Q, Li R, Qin J, Song L, Zhang Q, et al. LTF, PRTN3, and MNDA in synovial fluid as promising biomarkers for Periprosthetic joint infection: identification by quadrupole orbital-trap mass spectrometry. J Bone Joint Surg Am. 2019;101(24):2226-34. https://doi.org/10.2106/JBJS.18.01483.

17. Goswami K, Parvizi J, Maxwell CP. Current recommendations for the diagnosis of acute and chronic PJl for hip and knee-cell counts, alphadefensin, leukocyte esterase, next-generation sequencing. Curr Rev Musculoskelet Med. 2018;11(3):428-38. https://doi.org/10.1007/s12178-0189513-0.
18. Paziuk T, Rondon AJ, Goswami K, Tan TL, Parvizi J. A novel adjunct Indicator of Periprosthetic joint infection: platelet count and mean platelet volume. J Arthroplast. 2020;35(3):836-9. https://doi.org/10.1016/j.arth.2019.10.012.

19. Yuwen $P$, Chen $W, L v ~ H$, Feng C, Li Y, Zhang T, et al. Albumin and surgical site infection risk in orthopaedics: a meta-analysis. BMC Surg. 2017;17(1):7. https://doi.org/10.1186/s12893-016-0186-6.

20. Wu PP, Hsieh YP, Kor CT, Chiu PF. Association between albumin-globulin ratio and mortality in patients with chronic kidney disease. J Clin Med. 2019;8(11). https://doi.org/10.3390/jcm8111991.

21. Huang $W$, Sun $Y$, Xing $Y$, Wang $C$. Functional impairment and serum albumin predict in-hospital mortality in nonagenarians with acute infection: a retrospective cohort study. BMC Geriatr. 2019;19(1):269. https://doi.org/1 $0.1186 / s 12877-019-1301-1$

22. Mirsaeidi M, Omar HR, Sweiss N. Hypoalbuminemia is related to inflammation rather than malnutrition in sarcoidosis. Eur J Intern Med. 2018; 53:e14-6. https://doi.org/10.1016/j.ejim.2018.04.016.

23. Li K, Fu W, Bo Y, Zhu Y. Effect of albumin-globulin score and albumin to globulin ratio on survival in patients with heart failure: a retrospective cohort study in China. BMJ Open. 2018;8(7):e22960.

24. Guo HW, Yuan TZ, Chen JX, Zheng Y. Prognostic value of pretreatment albumin/globulin ratio in digestive system cancers: a meta-analysis. PLoS One. 2018;13(1):e189839.

25. Shimizu T, Ishizuka M, Suzuki T, Tanaka G, Park KH, Matsumoto T, et al. The preoperative globulin-to-albumin ratio, a novel inflammation-based prognostic system, predicts survival after potentially curative liver resection for patients with hepatocellular carcinoma. J Surg Oncol. 2017;116(8):116675. https://doi.org/10.1002/jso.24772.

26. Ukibe NR, Ndiuwem CK, Ogbu II, Ukibe SN, Ehiaghe FA, Ikimi CG. Prognostic value of some serum protein fractions as early index of clinical recovery in pulmonary tuberculosis subjects. Indian J Tuberc. 2020;67(2):167-71. https:// doi.org/10.1016/j.jitb.2019.08.015.

27. Xu H, Xie J, Yang J, Chen G, Huang Q, Pei F. Plasma fibrinogen and platelet count are referable tools for diagnosing Periprosthetic joint infection: a single-center retrospective cohort study. J Arthroplast. 2020;35(5):1361-7. https://doi.org/10.1016/j.arth.2019.12.015.

28. Tirumala V, Klemt C, Xiong L, Chen W, van den Kieboom J, Kwon YM. Diagnostic utility of platelet count/lymphocyte count ratio and platelet count/mean platelet volume ratio in periprosthetic joint infection following total knee arthroplasty. J Arthroplasty. 2020. https://doi.org/10.1016/j.arth.2 020.07.038.

29. Zhao Z, Liu J, Wang J, Xie T, Zhang Q, Feng S, et al. Platelet-to-lymphocyte ratio (PLR) and neutrophil-to-lymphocyte ratio (NLR) are associated with chronic hepatitis B virus (HBV) infection. Int Immunopharmacol. 2017;51:1-8. https://doi.org/10.1016/.intimp.2017.07.007.

30. Gasparyan AY, Ayvazyan L, Mukanova U, Yessirkepov M, Kitas GD. The platelet-to-lymphocyte ratio as an inflammatory marker in rheumatic diseases. Ann Lab Med. 2019:39(4):345-57. https://doi.org/10.3343/alm.201 9.39.4.345.

31. Meshaal MS, Nagi A, Eldamaty A, Elnaggar W, Gaber M, Rizk H. Neutrophilto-lymphocyte ratio (NLR) and platelet-to-lymphocyte ratio (PLR) as independent predictors of outcome in infective endocarditis (IE). Egypt Heart J. 2019;71(1):13. https://doi.org/10.1186/s43044-019-0014-2.

32. Parvizi J, Gehrke T. Definition of periprosthetic joint infection. J Arthroplast. 2014;29(7):1331. https://doi.org/10.1016/j.arth.2014.03.009.

33. Niedziela JT, Hudzik B, Szygula-Jurkiewicz B, Nowak JU, Polonski L, Gasior M, et al. Albumin-to-globulin ratio as an independent predictor of mortality in chronic heart failure. Biomark Med. 2018;12(7):749-57. https://doi.org/10.221 7/bmm-2017-0378.

34. Deng S, Gao J, Zhao Z, Tian M, Li Y, Gong Y. Albumin/Procalcitonin ratio is a sensitive early marker of nosocomial blood stream infection in patients with intra-cerebral hemorrhage. Surg Infect. 2019;20(8):643-9. https://doi.org/10.1 089/sur.2018.260.

35. Gupta D, Lis CG. Pretreatment serum albumin as a predictor of cancer survival: a systematic review of the epidemiological literature. Nutr J. 2010; 9(1):69. https://doi.org/10.1186/1475-2891-9-69.

36. Vincent JL, Dubois MJ, Navickis RJ, Wilkes MM. Hypoalbuminemia in acute illness: is there a rationale for intervention? A meta-analysis of cohort studies and controlled trials. Ann Surg. 2003;237(3):319-34. https://doi.org/1 0.1097/01.SLA.0000055547.93484.87.

37. Zhou T, Yu ST, Chen WZ, Xie R, Yu JC. Pretreatment albumin globulin ratio has a superior prognostic value in laryngeal squamous cell carcinoma 
patients: a comparison study. J Cancer. 2019;10(3):594-601. https://doi.org/1 0.7150/jca.28817.

38. Gabay C, Kushner I. Acute-phase proteins and other systemic responses to inflammation. N Engl J Med. 1999;340(6):448-54. https://doi.org/10.1056/ NEJM199902113400607.

39. Barbosa-Silva MC. Subjective and objective nutritional assessment methods: what do they really assess? Curr Opin Clin Nutr Metab Care. 2008;11(3):24854. https://doi.org/10.1097/MCO.0b013e3282fba5d7.

40. Green MD. Acute phase responses to novel, investigational vaccines in toxicology studies: the relationship between C-reactive protein and other acute phase proteins. Int J Toxicol. 2015;34(5):379-83. https://doi.org/10.11 77/1091581815598750.

41. Davalos D, Akassoglou K. Fibrinogen as a key regulator of inflammation in disease. Semin Immunopathol. 2012;34(1):43-62. https://doi.org/10.1007/ s00281-011-0290-8.

42. Jennewein C, Tran N, Paulus P, Ellinghaus P, Eble JA, Zacharowski K. Novel aspects of fibrin (ogen) fragments during inflammation. Mol Med. 2011; 17(5-6):568-73. https://doi.org/10.2119/molmed.2010.00146.

43. Li R, Shao HY, Hao LB, Yu BZ, Qu PF, Zhou YX, et al. Plasma fibrinogen exhibits better performance than plasma D-dimer in the diagnosis of Periprosthetic joint infection: a multicenter retrospective study. J Bone Joint Surg Am. 2019;101(7):613-9. https://doi.org/10.2106/JBJS.18.00624.

44. Xu H, Xie J, Huang Q, Lei Y, Zhang S, Pei F. Plasma fibrin degradation product and D-dimer are of limited value for diagnosing Periprosthetic joint infection. J Arthroplast. 2019;34(10):2454-60. https://doi.org/10.1016/j.arth.2 019.05.009.

45. Gaertner F, Ahmad Z, Rosenberger G, Fan S, Nicolai L, Busch B, et al. Migrating platelets are Mechano-scavengers that collect and bundle bacteria. Cell. 2017;171(6):1368-82. https://doi.org/10.1016/j.cell.2017.11.001.

46. Benito N, Franco M, Ribera A, Soriano A, Rodriguez-Pardo D, Sorli L, et al. Time trends in the aetiology of prosthetic joint infections: a multicentre cohort study. Clin Microbiol Infect. 2016;22(8):731-2.

\section{Publisher's Note}

Springer Nature remains neutral with regard to jurisdictional claims in published maps and institutional affiliations.

Ready to submit your research? Choose BMC and benefit from:

- fast, convenient online submission

- thorough peer review by experienced researchers in your field

- rapid publication on acceptance

- support for research data, including large and complex data types

- gold Open Access which fosters wider collaboration and increased citations

- maximum visibility for your research: over $100 \mathrm{M}$ website views per year

At $\mathrm{BMC}$, research is always in progress.

Learn more biomedcentral.com/submissions 